\title{
LA EVALUACIÓN EN LA EDUCACIÓN SUPERIOR: EL PROFESOR NOVEL Y SUS DIFICULTADES
}

\author{
AVALIAÇÃO DO ENSINO SUPERIOR: O PROFESSOR INICIANTE \\ E SUAS DIFICULDADES
}

\section{EVALUATION IN HIGHER EDUCATION: THE NOVICE TEACHER AND HIS DIFFICULTIES}

\section{Estefanía Fernández, Antón ${ }^{1}$}

\begin{abstract}
RESUMEN: Haciendo alusión a la evaluación formativa, comentamos las dificultades a las que hizo frente una profesora novel en el proceso de evaluación del alumnado universitario de segundo de Grado de Educación Primaria. Estas dificultades están narradas en forma de experiencia, y ésta tuvo lugar en una universidad de España, más específicamente, en una asignatura relacionada con los procedimientos metodológicos de investigación cualitativos y cuantitativos. Para recoger las dificultades del profesor novel, empleamos el diario de investigación durante un período de cuatro meses (febrero del año 2014 a junio del año 2014). Las dificultades que encontramos están relacionadas con la evaluación a lo largo del proceso, con los ítems de evaluación y con el examen.
\end{abstract}

PALABRAS-CLAVE: Evaluación. Educación Superior. Profesor novel.

RESUMO: No que concerne à avaliação formativa, discutimos as dificuldades de uma professora iniciante no processo de avaliação de estudantes universitários do curso no segundo semestre da Graduação em Formação de Professores para o Ensino Fundamental de uma universidade na Espanha. Estas dificuldades são narradas na forma de experiência, mais especificamente na disciplina relacionada com os procedimentos metodológicos de avaliação quali e quantivativa. Para coletar os dados relativos às dificuldades da professora iniciante, utilizamos um diário de investigação durante um período de quatro meses (fevereiro de 2014 a Junho de 2014). As dificuldades que encontramos estão relacionados com a avaliação ao longo do processo, com os itens que compõem a avaliação e com a prova.

PALAVRAS-CHAVE: Avaliação. Ensino Superior. Professor Iniciante.

ABSTRACT: Alluding to the formative evaluation, we discussed the difficulties of a novel teacher in the process of evaluation of university students in second grade of Primary Education. These difficulties are narrated in the form of experience, and this took place at a university in Spain, more specifically, on a subject of methodological procedures of qualitative and quantitative research. To collect the difficulties of novice teacher, we used the research journal for a period of four months (February 2014 to June 2014). The difficulties what we encounter are related with the evaluation process, with the evaluation items and with the exam.

KEY WORDS: Assessment. Higher Education. Noviceteacher.

\footnotetext{
${ }^{1}$ Doctoranda en Investigación e Innovación en Educación en la Universidad de Valladolid. Doctoranda en Educación y sociedad en la Universidad de Barcelona, Espanha. E-mail: fanitafernandez@ hotmail.es.

Recebido em: 22/06/2015 - Aprovado em: 03/11/2015.
} 


\section{RELATO DE EXPERIÊNCIA}

\section{INTRODUCCIÓN}

Desde este trabajo se presenta a la evaluación como un proceso clave para aumentar la motivacióndel alumnado por el aprendizaje. También se entiende que la evaluación es un proceso que ayuda al profesorado a aprender su función desde el análisis sistemático de sus actos.

El proceso de evaluación se ha entendido a lo largo de la historia de diferentes maneras. Al principio de los años XX la evaluación sólo valoraba los aprendizajes alcanzados por los alumnos que se pueden manifestar por medio de conductas. Entre 1940 y 1960 la evaluación estaba relacionada con el acto de juzgar. Y a partir de 1960 aparece la evaluación formativa de la que hablaremos posteriormente (DÍAZ, 2005).

Después de la anterior contextualización histórica, pasaremos a describirle concepto de evaluación en general y la evaluación formativa de manera más específica. Respecto a la evaluación, Tenbrink (2006, p.17) considera que "evaluar es asignar un valor a algo, juzgar. En educación, normalmente quiere decir juzgar a un estudiante, profesor o programa educativo".

Sanmartí (2007, p.28) define la evaluación de otra forma: "proceso de recogida y análisis de información destinado a describir la realidad, emitir juicios de valor y facilitar la toma de decisiones".

Siguiendo las indicaciones anteriores, la concepción que tenemos que tratar ahora es la de evaluación formativa, que de acuerdo a López (2006, p.103) “por evaluación formativa entendemos todo proceso de evaluación cuya finalidad principal es mejorar los procesos de aprendizaje y de enseñanza-aprendizaje que tienen lugar en un contexto educativo".

Este tipo de evaluación tiene las siguientes características (PUEYO; CLEMENTE; y LÓPEZ, 2009):

- La evaluación formativa no tiene como objetivo calificar al alumno, sino encontrar la mejor manera de ayudarle en su aprendizaje.

○ El alumnado aprende más y corrige sus errores.

- El docente aprende a realizar su trabajo de una manera más adecuada.

Otras características de la evaluación formativa son (ROSALES, 2003):

- Se aplican a lo largo del proceso. No es como la evaluación tradicional que tiene lugar al principio y al final del proceso. 


\section{RELATO DE EXPERIÊNCIA}

o La finalidad de esta evaluación es mejorar el proceso de enseñanza y aprendizaje.

o Intenta analizar la habilidad de cada alumnado en relación a cada aprendizaje.

Una vez familiarizados con los conceptos evaluación y evaluación formativa, queremos indicar el objetivo principal de este trabajo, que es: mostrar las dificultades de evaluación de una profesora novel en una asignatura del Grado de Educación Primaria y del curso puente de adaptación del Grado de Primaria. En las dificultades se hace mención de la evaluación formativa.

Aquí nos gustaría aclara que el profesor novel en el ámbito universitario es un profesional que lleva menos de tres años en la docencia universitaria. Para realizar sus funciones de enseñanza comienza con tareas como profesor becario, asociado o ayudante (FEIXAS, 2002).

Para terminar este apartado, queremos establecer que otros trabajos que analizan la evaluación en el contexto universitario son Barberá (2003); Barragán (2005); Biggs (2005); Pina (1996); Pueyo, et al. (2008); López (2012).

\section{CONTEXTUALIZACIÓN}

La experiencia que exponemos tuvo lugar en la Universidad de Valladolid, más específicamente, en la Facultad de Educación de Segovia (España).

La experiencia se narra desde la perspectiva de una alumna de la facultad que tuvo la oportunidad de ser profesora durante un cuatrimestre (febrero del 2014 al junio del 2014). Por lo tanto, el siguiente apartado, desarrollo de la experiencia, será presentado desde la perspectiva de una persona que fue docente novel en el ámbito universitario.

La asignatura que impartimos como profesores noveles estaba relacionada con los procedimientos metodológicos de investigación cualitativos y cuantitativos.

El alumnado del que hablaremos perteneció al segundo curso del Grado de Educación Primaria. Otros estudiantes cursaban el curso de adaptación al Grado de Educación Primaria. Los estudiantes estaban divididos en dos grupos: un grupo estaba constituido por 32 alumnos y el otro grupo por 62 alumnos.

Una vez que conocemos las características del contexto, nos gustaría señalar que los datos posteriores fueron recogidos en un diario de investigación. Un diario es una técnica de recogida de datos, en la cual persona investigadora recoge de manera sistemática sus impresiones, emociones, reflexiones y opiniones (ZABALZA, 2004). 


\section{RELATO DE EXPERIÊNCIA}

Los diarios se recogieron de manera continuada y después de cada clase. Además, los datos se repasaban cada semana para añadir aclaraciones que permitiesen entender con detalle las ideas. Los repasos semanales también fueron necesarios para comprobar si la información presentaba la realidad de manera objetiva o de manera subjetiva, es decir, influenciada por las emociones personales.

Los diarios contienen información sobre la evaluación a lo largo del proceso, sobre los ítems de evaluación, sobre el examen y sobre otras cuestiones que forman parte de otras investigaciones como el control emocional del profesor novel, su inseguridad, su relación con los estudiantes, etc.

Teniendo en cuenta las anteriores ideas, podemos apreciar que los diarios eran el resultado de las observaciones que realizábamos a los otros (alumnos), a nosotros mismos (autoobservación) y al proceso de enseñanza y aprendizaje

Las cuestiones que observábamos, y que aparecen en el párrafo anterior, fueron recogidas durante la semana previa a la función docente y durante los cuatro meses en los que desempeñamos nuestras tareas como profesores universitarios. Este período de tiempo comprende de febrero del año 2014 a junio del mismo año.

\section{DESARROLLO DE LA EXPERIENCIA}

La experiencia será comentada teniendo en cuenta tres temas: la evaluación a lo largo del proceso, los ítems de evaluación y el examen.

\section{La evaluación a lo largo del proceso}

El procedimiento de evaluación fue fijado durante los primeros días del segundo cuatrimestre; sin embargo, a medida que iban pasando las semanas, nos dimos cuenta de que nuestra forma de evaluación tenía carencias. Al principio pensábamos que nuestro procedimiento no tendría fallos tampoco imaginamos que desde las circunstancias íbamos a encontrar mejores formas de evaluar al alumnado.

También nos íbamos dando cuenta de que un buen sistema de evaluación podía ser uno de los elementos que influyen en la motivación del alumnado por la asignatura. Por ejemplo, dedicar un pequeño porcentaje de la nota final a la asistencia del alumnado no es tan efectivo como dedicar un pequeño porcentaje a la participación. Desde el primero sólo se consigue ocupar espacio en el aula y, desde el segundo procedimiento, se consiguen alumnos más preocupados por los contenidos académicos de cada asignatura.

Asimismo, echamos en falta procedimientos que aprendimos de los procesos de evaluación formativa en los que participábamos cuando éramos estudiantes de la diplomatura 


\section{RELATO DE EXPERIÊNCIA}

de Grado en Educación Infantil. Nos referimos a crear varias vías de evaluación: vía examen, vía examen y trabajo y vía más participativa en la que se incluyen trabajos, prácticas y examen. Dentro de cada vía hay unos porcentajes concretos para cada apartado a evaluar y, además, los estudiantes pueden elegir según sus circunstancias. Nosotros elegimos la misma vía para todos, estamos hablando de la vía examen y trabajo. La diferencia era que el alumnado que venía a clase tenía un porcentaje reservado por asistencia. Con este procedimiento la recompensa al trabajo entre los que asisten y no asisten al aula no es muy elevada y, por lo tanto, la motivación del alumnado por la asignatura disminuye.

Otro error fue dejar que los grupos de trabajo no fueran fijos desde las primeras semanas. En alguna ocasión, dejamos que algunos alumnos que no podían asistir al aula por motivos justificados formaran parte de grupos de trabajo consolidados. Esta forma de proceder dio lugar a que algunos alumnos descolgados no tuvieran la misma implicación en el desarrollo del trabajo escrito de la asignatura.

A pesar de que la evaluación formativa se lleva a cabo a lo largo de todo el proceso (ROSALES, 2003), hubiésemos preferido este sistema porque requiere de un proceso de valoración dividido en más pasos, y consideramos que esta división puede ser orientadora para un profesor novel.

\section{Los ítems de evaluación}

Cuando cursábamos las asignaturas de los antiguos planes de estudios (diplomatura), establecer ítems de evaluación para situaciones artificiales nos parecía una tarea sencilla. Ahora bien, seleccionar ítems para hacer una primera valoración de un trabajo escrito nos pareció muy complejo porque tuvimos en cuenta unos ítems en un principio, pero consideramos que faltaban ítems o que algunos se podían mejorar a lo largo del proceso.

La tarea de valorar un trabajo siguiendo unos ítems también nos resultó compleja porque en alguna ocasión evaluamos un trabajo olvidándonos de algunos ítems o añadíamos otros que no habíamos tenido en cuenta desde un principio. Así pues decidimos que todos los ítems de evaluación elegidos en un principio tenían que estar reflejados en los trabajos corregidos, pero que podíamos añadir comentarios de corrección con otros ítems siempre que estuvieran reflejados de manera detallada en el trabajo. Tomamos esta decisión porque nos parecía una buena forma de favorecer el aprendizaje de los estudiantes.

Incluso fue costoso valorar lo que se pedía en un ítem concreto y no algo parecido o cercano a la idea que se recogía dentro del ítem. 


\section{RELATO DE EXPERIÊNCIA}

\section{El examen}

Cuando sólo éramos estudiantes, el mejor examen para nosotros era el de desarrollo porque considerábamos que la prueba tipo test podía ser más complejas pues podía incluir preguntas complejas. No obstante, desde el rol del profesor, un examen con preguntas de desarrollo largas nos parecía demasiado complejo para ser evaluado porque es una prueba más subjetiva que los test. Así pues el modelo de nuestro examen combinaba las preguntas test con preguntas que requieren respuestas muy concretas.

Por otro lado, hemos de indicar que desde nuestro vida de estudiantes teníamos interiorizada la idea de que poner una nota en un examen u otra dependía del profesor, es decir, teníamos la creencia de que el examen era un instrumento para que el profesorado ejerciera poder sobre el alumnado. Esta convicción, que no es real, pero sí heredada al pertenecer al grupo estudiante durante muchos años, nos complicó el proceso de calificar un examen porque nos sentíamos culpables cada vez que aparecían notas excesivamente bajas. También aparecieron los miedos irracionales a las represalias de los estudiantes con notas bajas, ya que al no haber mucha diferencia de edad entre ellos y nosotros compartíamos muchos lugares públicos.

Otras dificultad añadida al instrumento examen es la argucia del estudiante; estamos hablando de la copia con sistemas no detectables fácilmente, el sistema de trampear las respuestas escribiendo lo peor posible para confundir al profesor, las excusas y suplicas desesperadas para aprobar la asignatura en la revisión del examen, etc.

\section{CONCLUSIONES}

En un principio, el profesor novel aprecia que un procedimiento de evaluación planificado no tenga carencias a lo largo del proceso; sin embargo, evaluar es una tarea que requiere de práctica y de análisis durante varios años y esto a su vez permite la mejora de la práctica docente. Pueyo et al. (2009) hacen referencia a la mejora de la función del profesorado con el empleo de la evaluación formativa.

El profesor novel también se da cuenta de que su procedimiento elegido desde sus criterios tiene diferentes fallos que influyen sobre la motivación del alumnado y sobre el buen funcionamiento de los grupos de trabajo. Según Biggs (2005), entre muchos otros, una buena evaluación mejora la motivación del alumnado por el aprendizaje.

Dentro del proceso de evaluación, el establecimiento de ítems de evaluación o la creación de la herramienta examen son otras acciones en las que el profesor novel encuentra dificultades. Los ítems tienen que ser utilizados para valorar, en otras palabras, una vez establecidos hay que emplearlos; aunque aparecen dificultades porque algunos de ellos se 


\section{RELATO DE EXPERIÊNCIA}

olvidan, se añaden otros nuevos y se valoran aproximaciones no lo que aparece definido exactamente en el ítem.

El profesor novel tiene interiorizadas las ideas que se asumen como verdaderas en el período de formación como alumnos; estamos hablando de las típicas creencias como son: el profesor me ha suspendido, si un profesor quiere te suspende haciendo uso del examen etc. Este tipo de ideas dificultan el acto de calificar al alumnado, ya que aparece un sentimiento de culpabilidad hacia las notas muy bajas y de miedo hacia las represalias del alumnado.

\section{REFERENCIAS}

BARBERÁ-GREGORI, Elena. Estado y tendencias de la evaluación en Educación Superior. Revista de Docencia Universitaria, La Rioja, ES, v. 3, n. 2. 2003. Disponible en: <http://goo.gl/Bxd5xW>. Acceso en: 22 junio 2015. ISSN 1887-4592.

BARRAGÁN, Raquel. El portafolio, metodología de evaluación y aprendizaje de cara al nuevo Espacio Europeo de Educación Superior: una experiencia práctica en la Universidad de Sevilla. Revista Latinoamericana de Tecnología Educativa, Sevilla, ES, v. 4, n.1, p. 121140. 2005. Disponible en: 〈http://goo.gl/s5yqeZ〉. Acceso en: 22 junio 2015.

ISSN-e 1695-288X.

BIGGS, John. Calidad del aprendizaje universitario. Madrid: Narcea, 2005.

COLL, Cesar; ROCHERA, María José; MAYORDOMO, Rosa María; NARANJO, Mila. Evaluación continua y ayuda al aprendizaje. Análisis de una experiencia de innovación en Educación Superior con apoyo de las TIC. Revista Electrónica de Investigación Psicoeducativa, ES, v. 5 (3), n. 13, p. 783-804. 2007. Disponible en: <http://goo.gl/Oc4E9T >. Acceso en: 22 junio 2015. ISSN 1696-2095.

DÍAZ, Jordi. La evaluación formativa como instrumento de aprendizaje en educación física. Zaragoza: Inde, 2005.

FEIXAS, Mónica. El profesorado novel: estudio de su problemática en la Universitat Autónoma de Barcelona. Revista de Docencia Universitaria, La Rioja, ES, v. 2, n. 1. 2002. Disponible en: 〈http://goo.gl/VU8jWm> . Acceso en: 22 junio 2015. ISSN 1887-4592.

LÓPEZ, Víctor. El papel de la evaluación formativa en el proceso de convergencia hacia el EEES. Análisis del estado de la cuestión y presentación de un sistema de intervención.

Revista Interuniversitaria de Formación del Profesorado, La Rioja, ES, v. 20, p. 93-120. 2006. Disponible en: 〈http://goo.gl/pMVX29>. Acceso en: 22 junio 2015. ISSN 0213-8464.

LÓPEZ, Víctor. Evaluación formativa y compartida en la universidad: clarificación de conceptos y propuestas de intervención desde la Red Interuniversitaria de Evaluación Formativa. Psychology, Society, \& Education, v. 4, n. 1, p. 117-130. 2012. Disponible en: 〈http://goo.gl/MG7B6D〉. Acceso en: 22 junio 2015. ISSN 1989-709X. 
PINA, Fuensanta. La evaluación de los alumnos en el contexto de la evaluación de la calidad de las universidades. Revista de Investigación Educativa, RIE, v. 14, n. 2, p. 25-50. 1996. Disponible en: <http://goo.gl/a6RJX4>. Acceso en: 22 junio 2015. ISSN 0212-4068.

PÉREZ, Ángel; CLEMENTE, Julián; LÓPEZ, Víctor. Evaluación formativa y compartida en el Espacio Europeo de Educación Superior (EEES). En: LÓPEZ, Víctor (Coord.).

Evaluación formativa y compartida en Educación Superior: propuestas, técnicas, instrumentos y experiencias. Madrid: Narcea, 2009, p. 19-45.

PÉREZ, Ángel et al. Evaluación formativa y compartida en la docencia universitaria y el Espacio Europeo de Educación Superior: cuestiones clave para su puesta en práctica. Revista de Educación, v. 347, p. 435-451, sep./dic. 2008. Disponible en: 〈 http://goo.gl/pHohX9>. Acceso en: 22 junio 2015. ISSN 1988-592X.

ROSALES, Carlos. Criterios para una evaluación formativa. Madrid: Narcea, 2003.

SANMARTÍ, Neus. 10 ideas claves. Evaluar para aprender. Barcelona: Graó, 2007.

TENBRINK, Terry. Evaluación guía práctica para profesores. Madrid: Narcea, 2006.

ZABALZA, Miguel Ángel. Diarios de clase: un instrumento de investigación y desarrollo profesional. Madrid: Narcea, 2004.

Como citar este documento:

FERNÁNDEZ ANTÓN, Estefanía. La evaluación en la Educación Superior: el profesor novel y sus dificultades. ETD - Educação Temática Digital, Campinas, SP, v. 17, n. 3, set./nov. 2015. ISSN 1676-2592. Disponível em: 〈http://periodicos.sbu.unicamp.br/ojs/index.php/etd/article/view/8637361〉. Acesso em: $14 \mathrm{dez}$. 2015. 